\title{
Compression of Pseudo-periodic Signals Using 2D Wavelet Transform
}

\author{
Dominic Mathew ${ }^{1, *}$, V.P. Devassia ${ }^{2}$, Tessamma Thomas ${ }^{3}$ \\ ${ }^{1}$ Department of Applied Electronics \& Instrumentation, Rajagiri School of Engineering \& Technology, M.G. University \\ Kakkanad, Cochin, Kerala, Pin , 682039, India \\ ${ }^{2}$ College of Engineering, Cochin University, Chengannur, Kerala, Pin , 689121, India \\ ${ }^{3}$ Department of Electronics, Cochin University, CUSAT, Cochin, Kerala, Pin , 682022, India
}

\begin{abstract}
An improved method to compress of pseudo-periodic 1-dimensional signals like voiced speech, music, ECG etc is suggested. The pitch synchronous property of such signals is utilized to increase the efficiency of compression, to minimize losses and thus to enhance the quality of the reconstruction. Results show higher signal to noise ratio, higher compression ratio and lower percentage distortion with the new method of 2-D compression as compared to 1-D compression. A new method employing the k-means clustering algorithm is used to determine the periodicity of signals.
\end{abstract}

Keywords Pitch-synchronous, 2-D Wavelet Transform, K- means Clustering, Compression

\section{Introduction}

Generally for any signal compression method larger compression ratios result in higher signal losses, leading to poorer quality of the reconstructed signal. Signals like voiced speech, music ECG etc, exhibit oscillatory nature[1]. These signals though not perfectly periodic, can be classified as pseudo-periodic. Once its periodicity is known the signal can be represented in a 2-D form and decomposed using two-dimensional wavelets. Wavelet transform methods have proved to be very powerful techniques for signal compression. The signal represented in 2-D form and transformed into the time-frequency domain is well suited for detection and removal of redundancies. In this paper a new algorithm is proposed to minimize losses and to improve compression efficiency using 2-D wavelet transform. The study was conducted on speech, music and ECG signals. A novel and fast method employing the k-means clustering algorithm is used for extracting the periodicity of signals.

\section{The Proposed Method}

The proposed method consists of the following steps 1) Pre-processing, 2) Pitch-synchronous representation, 3) 2D wavelet decomposition, 4) Reduction of the total number of wavelet coefficients.

The quality of reconstruction with the proposed compres-

* Corresponding author:

dominic_m73@yahoo.com (Dominic Mathew)

Published online at http://journal.sapub.org/ajsp

Copyright (C) 2011 Scientific \& Academic Publishing. All Rights Reserved sion method is compared with that of the $1 \mathrm{D}$ wavelet compression method. Figures 1 to 7 show the evaluation results of such comparisons. The samples of reconstruction for various types of signals are shown in Figures 8 to 11. The wavelet used for decomposition is $\mathrm{db} 2$, due to its simplicity. For speech signal decomposition a maximum scale of up to 5 is adequate[2]. For ECG and music signals a maximum decomposition level of up to 4 is used for both 2D and 1D compression.

\subsection{Pre-processing}

A sampling frequency of $8 \mathrm{KHz}$ is used for the signals. It is then filtered using a 2nd order butter-worth filter to remove the high frequency hum and noise. It is then amplitude normalized to eliminate the effects in signal characteristics recorded under various conditions

The pitch peaks are detected using the k-means clustering algorithm[3]. The statistical approach of clustering eliminates the discrepancies of pitch period measurements due to pseudo-periodicity of the signals. Thus it helps to filter out the dominant peaks in the signal samples and eliminates the non-relevant ones. The squared Euclidean distance method is used as the criterion for clustering since it is the simplest and fastest. The Euclidean distance measure is given by the following equation

$$
d_{2}\left(\mathbf{x}_{i,} \mathbf{x}_{j}\right)=\sqrt{\sum_{k=1}^{d}\left(x_{i, k}-x_{j, k}\right)^{2}}=\left\|\mathbf{x}_{i}-\mathbf{x}_{j}\right\|_{2}
$$

where $\mathbf{x}$ is a single data vector used for clustering, $i$ and $j$ are the $i^{\text {th }}$ and $j^{\text {th }}$ data vectors (patterns) and $d$ is the dimensionality of the data vector[4].

The dominant peaks in the signal determine the pitch periods. The number of signal samples between the peaks 
denotes the pitch period length.

\subsection{Pitch -synchronous Representation}

Once the period between the peaks are known, a pseudoperiodic signal $\mathbf{s}(n)$ with $N$ samples can be assumed to be a sequence of $P(k)$ pitch periods [1]. Each period length is stored in a vector $\mathbf{v}(k)=\left[v_{0}(k), v_{1}(k), \ldots v_{P(k)-1}(k)\right]^{\mathrm{T}}$. The vector components $\mathbf{v}_{q}(k)$ can be expressed in terms of the signal $s(n)$ as follows

$$
\mathbf{v}_{q}(k)=s\left(q+\sum_{r=0}^{k-1} P(r)\right), q=0,1, \ldots \mathrm{P}(k)
$$

where $q$ and $k$ are referred to as the inter-period and the period-count indices respectively. This $2 \mathrm{D}$ form of the $1 \mathrm{D}$ signal is referred to as the pitch-synchronous (PS) representation. The 2D matrix representation of $q$-rows and $k$ columns enables us to perform 2D wavelet analysis and decomposition for compressing the signal.

\subsection{Two-Dimensional Wavelet Decomposition}

The 2D discrete wavelet decomposition of the PS form can be represented as sum of coarse resolution (level $J$ ) approximation coefficients and a sum of fine to coarse resolution (levels 1 to $J$ ) detail coefficients[5]. There are 3 types of detail coefficients and basis functions: vertical, horizontal and diagonal details. The transform coefficients are given approximately by the integrals

$$
\begin{aligned}
& a_{J, m, n} \approx \iint \Phi_{J, m, n}(x, y) s(x, y) d x d y \\
& d_{j, m, n}^{v} \approx \iint \Psi_{j, m, n}^{v}(x, y) s(x, y) d x d y \\
& d_{j, m, n}^{h} \approx \iint \Psi_{j, m, n}^{h}(x, y) s(x, y) d x d y \\
& d^{d}{ }_{j, m, n} \approx \iint \Psi_{j, m, n}^{d}(x, y) s(x, y) d x d y
\end{aligned}
$$

where $a_{J, m, n}$ represents the approximation coefficients at level $J$ and $d_{j, m, n}^{v}, d_{j, m, n}^{h}, d^{d}{ }_{j, m, n}$ are the vertical, horizontal and diagonal detail coefficients respectively. The 2D basis functions are generated from the father wavelet $\Phi$ and mother wavelets $\Psi^{v}, \Psi^{h}$ and $\Psi^{d}$ by scaling and translation as follows

$$
\begin{aligned}
& \Phi_{J, m, n}(x, y)=2^{-J} \Phi\left(2^{-J} x-m, 2^{-J} y-n\right) \\
& \Psi_{j, m, n}^{v}(x, y)=2^{-j} \Psi^{v}\left(2^{-j} x-m, 2^{-j} y-n\right) \\
& \Psi_{j, m, n}^{h}(x, y)=2^{-j} \Psi^{h}\left(2^{-j} x-m, 2^{-j} y-n\right) \\
& \Psi_{j, m, n}^{d}(x, y)=2^{-j} \Psi^{d}\left(2^{-j} x-m, 2^{-j} y-n\right)
\end{aligned}
$$

The 2D wavelet analysis of PS representation of the 1D signal can be considered a multi-resolutional image analysis[6]. Multi-resolutional analysis is a simultaneous representation on different threshold levels. This can be considered the output of successive convolution operations on an input sequence with high-pass and low-pass filters[7]. The impulse response of these high and low pass filters are represented separately.

The 2-D data matrix is decomposed into four separable bands. This results in four transform components consisting of approximation (low-low), horizontal details (low-high), vertical details (high-low) and diagonal details (high-high) for each resolution level. The decomposition operations are repeated on the low-low band in each level to compute the wavelet transform at the subsequent level. Due to the dyadic nature of discrete wavelet transforms the output is down-sampled by two in each stage.

\subsection{Reduction of Transform Coefficients}

The wavelet transform coefficients are represented in 2-D matrix. For any level of decomposition the number of column vectors in each sub-band is represented by $J, d s(i)$ represents the vector coefficient with index $i$ and $I$ represents the length of each vector. Reduction in the number of transform coefficients is achieved using the following algorithm:

A. For $J$ column vectors of approximate and detailed coefficients, the energy of the vector is compared with a threshold ET. If the vector energy is less than ET then all coefficients in that vector is set to zero.

$$
\begin{aligned}
& \text { for } j=1 \text { to } J \\
& \text { if } \sum_{i=1}^{I} d s(i)^{2}<E T \\
& \text { for } i=1 \text { to } I ; d s(i)=0 ; \\
& \text { end; end; end; }
\end{aligned}
$$

This is justified since the contribution of low energy transform vectors in reconstructing the original signal is insignificant.

B. At every level of decomposition the first $(j=1)$ column vector in each sub-band is retained. The Euclidean distance between any two vectors of equal length is a measure of the similarity/dissimilarity between them. Therefore

$$
\begin{aligned}
& \text { for } j=1 \text { to } J \\
& \text { for } m=2 \text { to } J \& m \neq j \\
& \text { if } \sum_{i=1}^{I}\left[d s(i)_{j}-d s(i)_{m}\right]^{2}<S F \\
& \text { for } i=1 \text { to } I \\
& d s(i)_{m}=d s(i)_{j} ; \\
& \text { end; end; end; end; }
\end{aligned}
$$

where $S F$ is a similarity factor. The values of $j$ and $m$ are stored for transmission. During reconstruction the integers $j$ and $m$ are used to restore the $m^{\text {th }}$ vector with the coefficients of $j^{\text {th }}$ vector.

C. If the absolute vector coefficients values are less than a magnitude threshold $T H$, then it is set to zero

$$
\begin{aligned}
& \text { for } j=1 \text { to } J \\
& \text { for } i=1 \text { to } I \\
& \text { if }|d s(i)|<T H, d s(i)=0 \text {; } \\
& \text { end; end; end; }
\end{aligned}
$$




\section{Evaluation of Reconstructed Signal}

The quality of the reconstructed signal is evaluated using standard measures viz. the signal to noise ratio (SNR) and percentage root mean square difference $(P R D)$ as well as the mean opinion score $(M O S)$. The measure of compression is obtained from the compression ratio $(C R)$, is defined as the ratio of the number of the retained coefficients after applying the threshold algorithm to number of the coefficients in the original signal.

\subsection{Signal to Noise Ratio}

Signal to noise ratio $(S N R)$ is given by the equation

$$
S N R=10 * \log _{10} \frac{\sum_{n=1}^{N}[s(n)-\bar{s}]^{2}}{\sum_{n=1}^{N}[e(n)-\bar{e}]^{2}}
$$

where $s(n)$ is $n^{\text {th }}$ sample of the original signal, $\bar{s}$ is the mean of the $\mathrm{N}$ signal samples, $\mathrm{N}$ is the length of signal, $e(n)$ is the error between the original signal and reconstructed signal, given by $s(n)-s_{r}(n)$, and $\bar{e}$ is the mean of the error signal over $\mathrm{N}$ samples.

\subsection{Percentage Root Mean Square Difference}

Percentage root mean square difference $(P R D)$ is defined by the following equation,

$$
P R D=100 * \sqrt{\frac{\sum_{n=1}^{N}\left[s(n)-s_{r}(n)\right]^{2}}{\sum_{n=1}^{N}[s(n)-\bar{s}]^{2}}}
$$

where $s_{r}(n)$ is the $n^{\text {th }}$ sample of the reconstructed signal.

\subsection{Mean Opinion Score}

The mean opinion score $(M O S)$ is calculated by taking the arithmetic mean of the perceived listening quality expressed in terms of scores ranging from 5,4,3,2 and 1 for excellent, good, fair, poor and unsatisfactory respectively. Despite the significant advances in modern measuring and evaluation technology the human ear alone is able to judge the aesthetic or artistic quality of sound[8,9].

\section{Results and Conclusions}

The proposed method was tested on various voiced signal segments selected from speech databases ELSDSR and TIMIT, ECG signals from database MIT-BIH and musical instrument signals like Guitar and Flute. Figures 1 up to 4 show the values of PRD and SNR values obtained on various signal segments with a compression ratio equal to 12 . Fig-1 \& 2 shows the results of the study done on segments of Male and Female speech signals respectively from the ELSDSR database. It compares the performance of the proposed 2-D compression algorithm, with that of 1-D wavelet decomposition method. ELSDSR Corpus is provided by the Depart- ment of Informatics and Mathematical Modelling, Technical University of Denmark. Fig-3 shows results obtained for Female (F1 \& F2) and Male (M1 \& M2) speech signals from TIMIT. The TIMIT Speech Corpus is provided by DARPA. Fig-4 shows the results for ECG signal - Record 12247-01 from the Massachusetts Institute of Technology and the Beth Israel Hospital (MIT_BIH) compression database and also on typical recorded notes of Guitar and Flute. Fig $5 \& 6$ shows the variation of $P R D$ and $S N R$ values for different values of $C R$ on speech segment F2 of TIMIT. It clearly shows that the rate of change of $P R D$ and $S N R$ with respect to $C R$ is much lower for the $2 \mathrm{D}$ algorithm as compared to $1 \mathrm{D}$ algorithm. Figure-7 shows the variation of average $M O S$ values for different values of $C R$ for both $2 \mathrm{D}$ and $1 \mathrm{D}$ algorithms. The $C R$ values of 5, 10 and 15 were selected so that the differences in quality of the audio signal can be easily perceived.

Figs- 8 up to 11 show typical segments of the reconstructed signals along with the original signals for various signals with compression ratio of $12: 1$ for both the 2-D and 1-D compression methods. The original signal is plotted in solid dark line. The reconstructed signal is plotted in dashed red line. Results indicate that the performance of the proposed $2 \mathrm{D}$ compression algorithm is more efficient compared to that of compression using 1-D method. For similar PRD and $S N R$ values the $2 \mathrm{D}$ method gives higher compression ratios. The proposed 2-D compression method also offers better quality of reconstruction for the same compression ratios over 1-D method. Similarly it is seen that the new method offers better compression ratios if the quality of reconstructed signal is maintained the same as that of 1-D method.
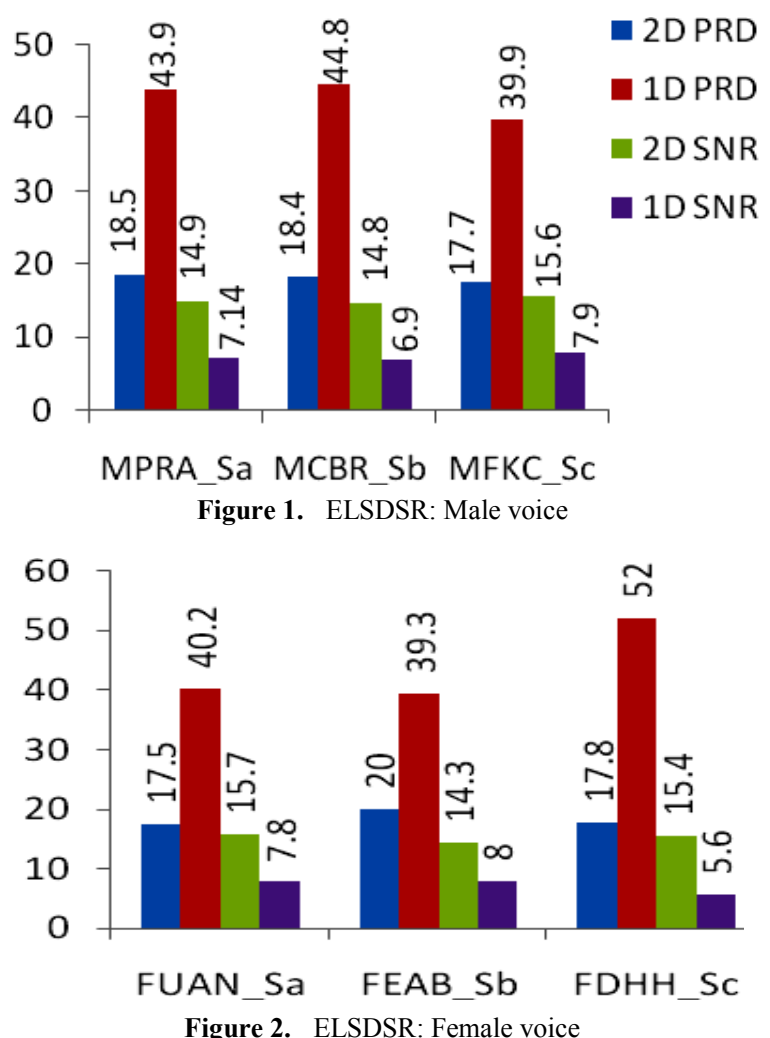


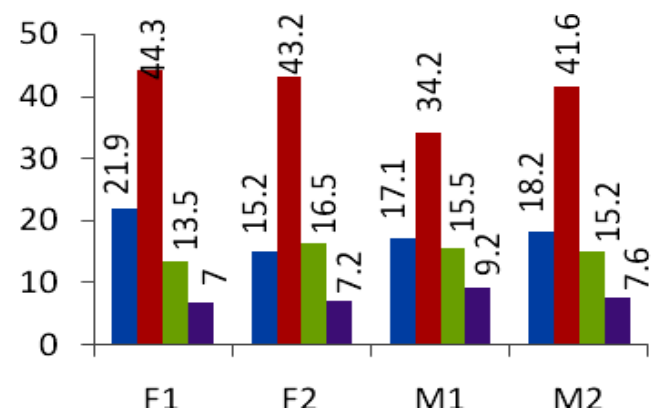

Figure 3. TIMIT: F1,F2 - Female, M1,M2 - Male

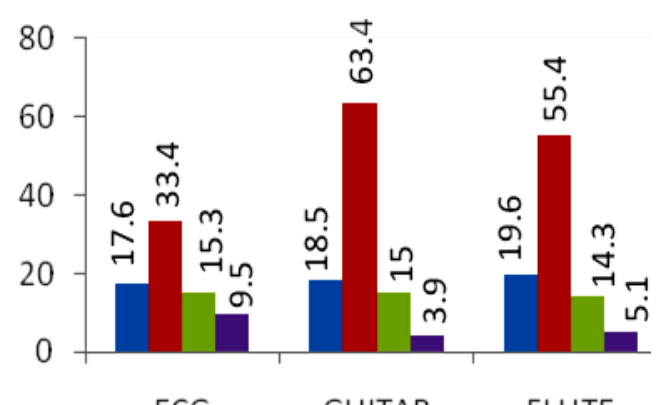

ECG GUITAR FLUTE

Figure 4. ECG \& Musical Instruments

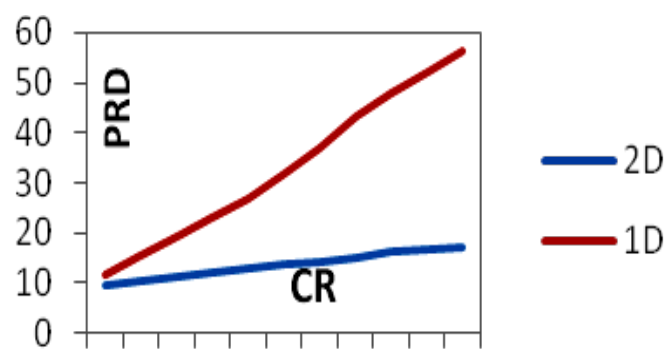

$\begin{array}{llllll}5 & 7 & 9 & 11 & 13 & 15\end{array}$

Figure 5. PRD vs. CR TIMIT (F2)

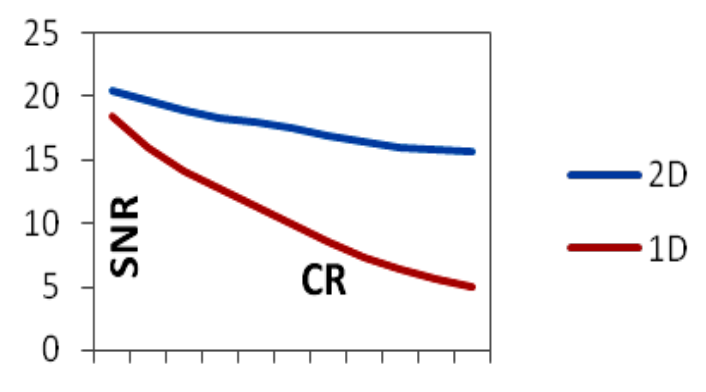

$\begin{array}{llllll}5 & 7 & 9 & 11 & 13 & 15\end{array}$

Figure 6. SNR vs. CR TIMIT (F2)

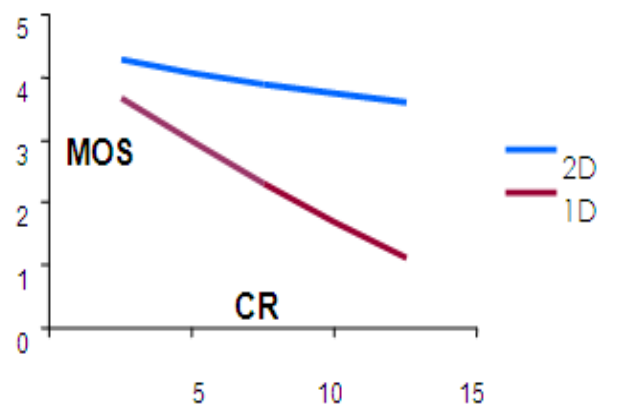

Figure 7. $\mathrm{MOS}$ vs $\mathrm{CR}$
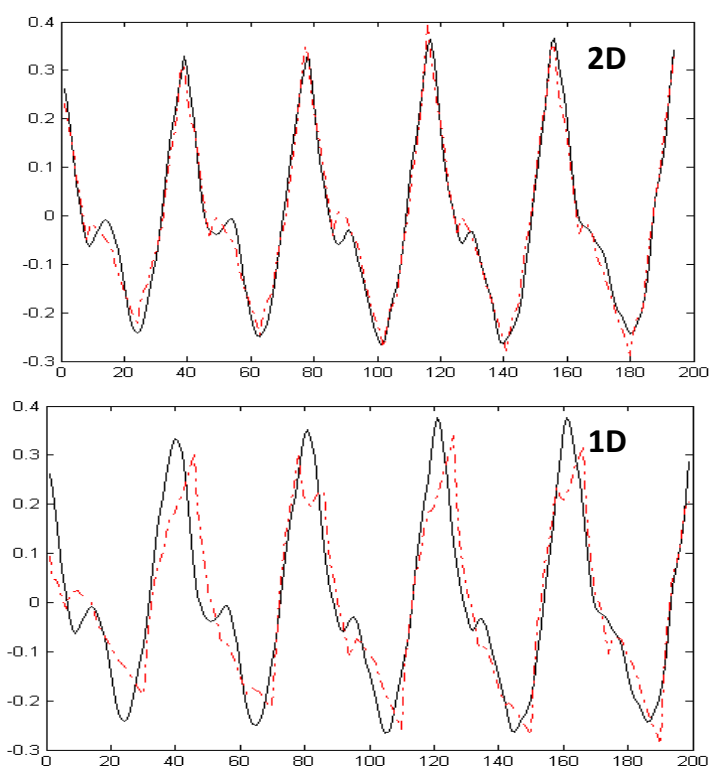

Figure 8. FEAB $\mathrm{Sb}$
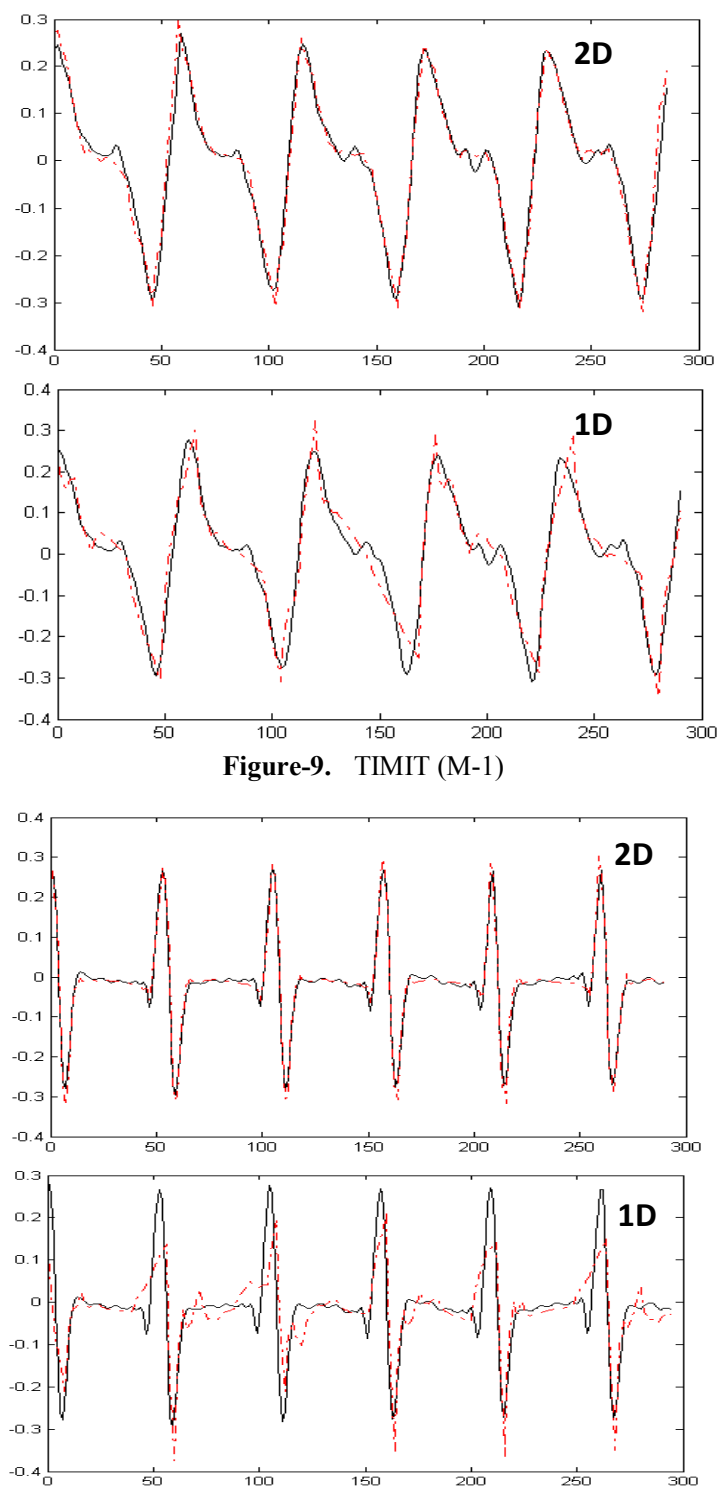

Figure 10. GUITAR 

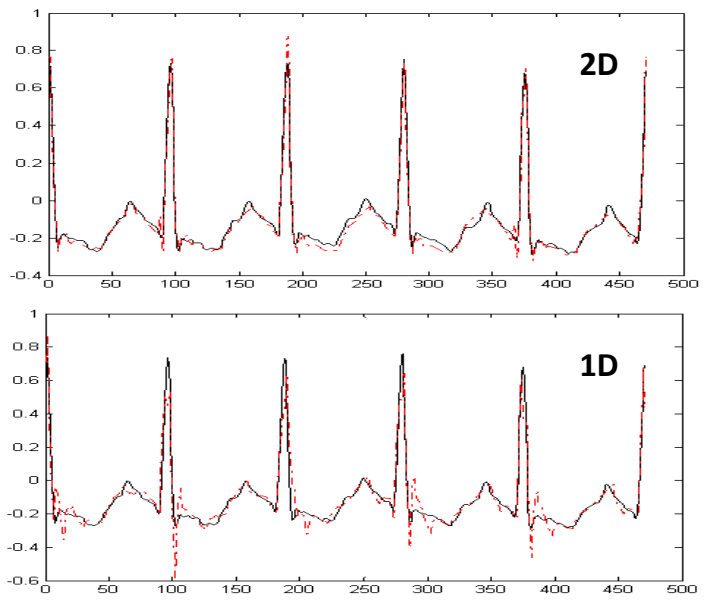

Figure 11. ECG - 1224701 (MIT-BIH)

\section{REFERENCES}

[1] Gianopaolo Evangelista, "Pitch-Synchronous Wavelet Representations of Speech and Music Signals", IEEE Trans On Signal Processing", vol.-41, No.12, December 1993, $3313-3330$

[2] J.I. Agbinya, "Discrete Wavelet Transform Techniques in Speech processing", IEEE Tencon Digital Signal Processing
Applications Proceedings, New York, 1996, 514-519

[3] Dominic Mathew, V.P. Devassia, Tessamma Thomas, “A $k$-means Clustering Algorithm for Pitch Frequency Estimation and Classification of Speech Signals", Proceedings of the IEEE ICSIP, vol. 1, Macmillan India Ltd, Hubli, 7-9 December 2006, 967-972

[4] A.K. Jain, M.N. Murthy, P.J. Flynn, "Data Clustering: A Review", ACM Computing Surveys, vol. 31, No.3, September 1999, 264-323

[5] Andres Bruce and Hong-Ye Gao, Applied Wavelet Analysis with S-PLUS, Springer-Verlag, New York, 1996

[6] S.G. Mallat, "A Theory of Multiresolutional Signal decomposition: The wavelet representation", IEEE Trans of Patt. Analysis and Mach. Intell, vol. 11, July 1989, 674-693

[7] S.G. Mallat, "Multifrequency channel decomposition of images and wavelet models", IEEE Trans of Acoust, Speech, Signal Processing, vol. 37, Dec 1989, 2091 - 2110

[8] W. Hoeg (Deutsch Telekom Berkom), L. Christensen (Danmarks Radio) R. Walker (BBC), "Subjective assessment of audio quality - the means and methods within EBU", EBU Technical Review, Winter 1997

[9] Huber R, Kollmeier B, "PEMO-Q-A New Method for Objective Audio Quality Assessment Using a Model of Auditory Perception", IEEE Trans of Acoust, Speech, Signal Processing, vol. 14, Nov 2006, 1902 - 1911 